\section{A new method of biofeedback in the management of low
vision management of low
vision}

Department of

Ophthalmology

(Director: Prof Corrado

Balacco Gabrieli)

University of Rome

'La Sapienza'

Rome, Italy

Correspondence:

MT Contestabile

Istituto di Oftalmologia

Università di Roma 'La

Sapienza'

Policlinico Umberto I

Roma 00161, Italy

Tel: Italy 06490296

Fax: Italy 064457706

E-mail: corrado.balacco@

uniroma1.it

Received: 6 July 2000

Accepted in revised form: 1 June 2001

\begin{abstract}
Twenty-eight patients with low vision were enrolled into the study. A preliminary study was carried out on 18 eyes of 13 patients with low vision who underwent visual rehabilitation with a new instrument for biofeedback (BF) applied to vision; improved biofeedback integrated system (Ibis). Successively, eight patients (16 eyes) with bilateral low vision were subjected to biofeedback in one eye. The experimental and control eye were evaluated separately. Then a placebo training was developed on seven patients (12 eyes). Visual acuity, colour vision, automated perimetry, contrast sensitivity and flash VEP were evaluated. A brief review of the literature and the possible mechanisms behind the results are discussed.

Eye (2002) 16, 472-480. doi:10.1038/

s.eye. 6700046
\end{abstract}

Keywords: biofeedback applied to vision; low vision

\section{Introduction}

Techniques of biofeedback (BF) are used to obtain control of involuntary functions. Through these methods, adopted in various branches of medicine, the patient learns in successive stages to: (a) appreciate the variations of a bodily function through a system that measures and converts these in acoustic and/or luminous signals; (b) modify these signals and, therefore, the function connected to them; (c) automatically control the function through practice even in the absence of the return signal.

There are two types of biofeedback; direct and indirect. In the former the patient can directly measure the bodily function or process to be controlled, for instance, to
MT Contestabile, SM Recupero, D Palladino, M De Stefanis, S Abdolrahimzadeh, F Suppressa and C Balacco Gabrieli appreciate muscular tension through an electromyograph connected to an appropriate device. On the other hand, indirect BF is when the patient cannot directly evaluate the bodily function but has to control this by monitoring a connected activity. An example of this is learning to sense emotional alterations through changes in perspiration.

BF applied to vision is still being studied both in its methodological and physiological aspects. Some instruments have been used in the past; initially in the treatment of myopia and then in other ocular pathologies. ${ }^{1-8}$ However, at present knowledge regarding BF applied to vision is very poor.

The purpose of the current study was to investigate the effect of biofeedback applied to vision with a new instrument for visual training in patients with low vision. We first conducted a preliminary investigation. Successively, on the basis of the results obtained we extended the research in order to further investigate the efficacy of the procedure. Then a placebo training was developed to mimic the technique of biofeedback experimented.

\section{Materials and methods}

Twenty-eight patients with low vision were enrolled in the study according to the WHO classification (International Classification of Diseases, WHO, 1977). Eighteen eyes of 13 patients with low vision were enrolled into the first part of our study. This part of the investigation was a preliminary study. All patients had received pharmacological treatment in the past based on the specific pathology in each case which eventually led to low vision. There were five bilateral and eight unilateral cases. The patients were composed of six males and seven females with an age range of 30-75 years. The criteria chosen for inclusion in the study protocol 
were visual acuity of 3/10 or less, stable for at least one year before training, advanced macular or optic nerve pathology, no history of epilepsy and good patient collaboration.

Seven patients (nine eyes) had age-related macular degeneration, two patients (two eyes) had myopic maculopathy and four patients (seven eyes) had diffuse pallor of the optic nerve. All patients underwent visual training with infrared photo-stimulation using a new instrument for BF; improved biofeedback integrated system (Ibis).

Ibis is composed of an electronic and an optical unit shown in detail in Figure 1. Training was performed in a darkened room. During each session, after having aligned the patient's eye, the instrument was switched on, thus starting infrared photostimulation and also foveal flicker stimulation. The latter was performed by turning on the internal foveal stimulator consisting of a red intermittent light inside the eyepiece. The intensity of this foveal stimulus was chosen based on the visual acuity in each case. The frequency was set at the value immediately lower than that at which the patient could see a fixed red light (critical fusion frequency). Thus, the volume switch was turned on by the operator. The patient was initially asked to look at the light inside the eyepiece. Thus, the operator made fine movements of the joystick to help the patient find the position at which the auditory signal reached the highest volume. Then the patient had to maintain this volume which corresponded to the highest level of BF. The increase in volume was shown both by the digital display of information and the rise of the peak on the monitor. Each patient was trained twice a week for a total of 15 sessions. Every session involved three applications

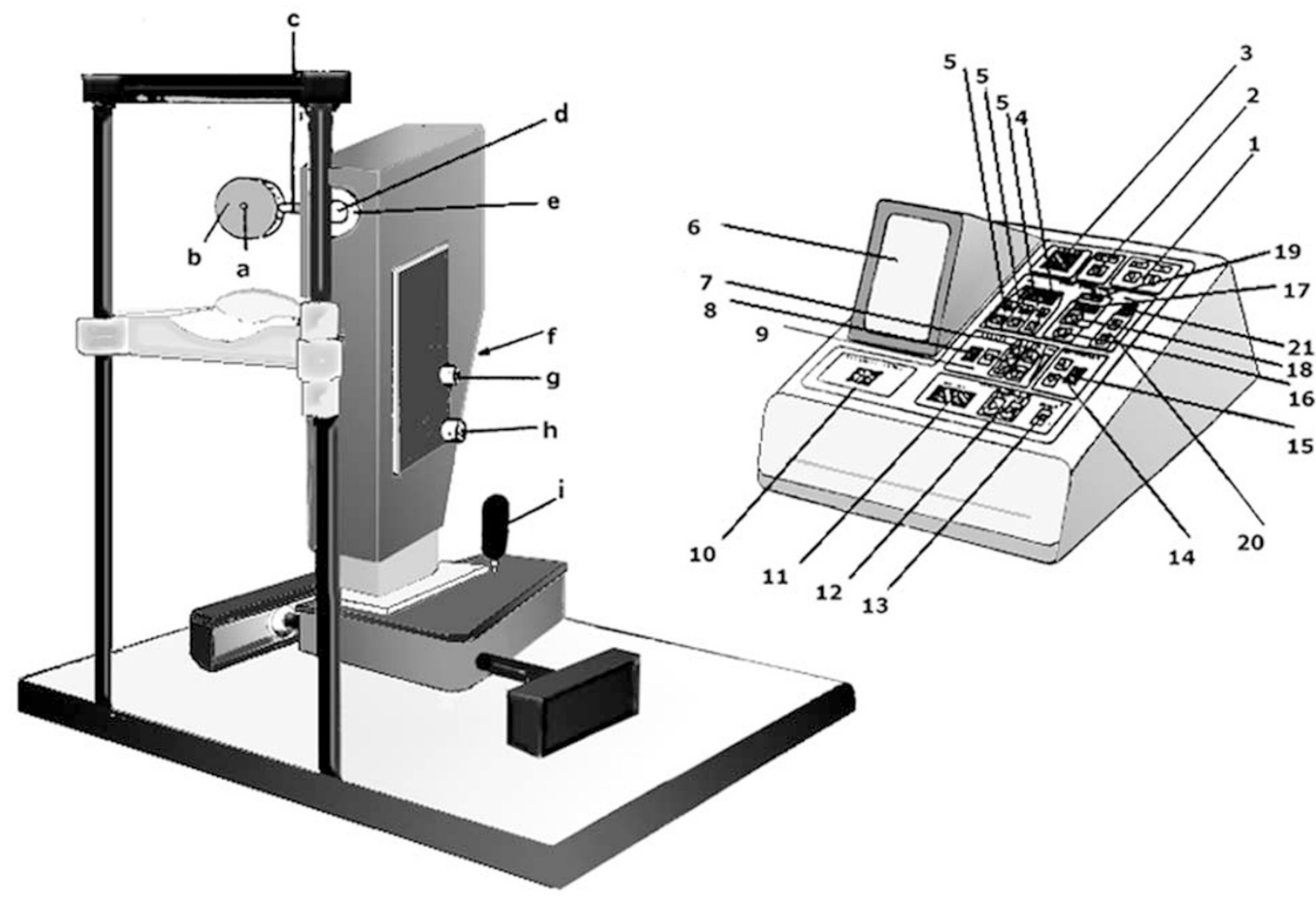

Figure 1 Improved biofeedback integrated system (Ibis). Electronic Unit: 1, On/Off switch; 2, switch for alignment led; 3, indicator of intensity of enivironment light; 4 , indicator of period of intermittence of foveal stimulus; 5 , selectors of period of intermittence of foveal stimulus; 6, monitor to control alignment, level of BF, direction of gaze; 7, regulator of the volume of feedback sound (headset); 8, regulator of the volume of feedback sound (environment); 9, selector/indicator of feedback sound; 10, digital indicator of feedback level; 11, timer; 12, commands for timer; 13, timer reset; 14, selector of background light of internal fixation point; 15, indicator of background light of internal fixation point; 16, switch for external foveal stimulator; 17, indicator light for external foveal stimulator; 18, switch for internal foveal stimulator; 19, indicator light for internal foveal stimulator; 20, selector of foveal stimulus intensity; 21, indicator of luminous intensity of foveal stimulus. Optical Unit: $a$, terminal led of external foveal stimulator; b, occluder; c, telescopic regulator of interpupillary distance; d, internal foveal stimulator; e, eyepiece; f, electronic segment connection socket; $\mathrm{g}$, regulator of vertical target for accommodation request; $\mathrm{h}$, regulator of horizontal target for accommodation request. 
lasting 3 min each with a brief pause in between. Refraction was performed in a standardized fashion and visual acuity was tested at a distance of 5 meters using standard Snellen charts. Best corrected visual acuity was expressed in decimal notation. Visual acuity was measured after every three sessions using the same charts. At the last follow-up a visual acuity chart with a different arrangement of letters was used. All patients were subjected to a series of tests before and after having completed treatment. The number of words read per minute was calculated using letters with a size corresponding to the second character of a near visual acuity chart (Distance $\mathrm{D}=0.75$ ). The height of the letters on this chart subtend an angle of $5 \mathrm{~min}$ at a distance of $0.75 \mathrm{~cm}$. A video for low vision provided with various enlargement levels for the letters was used. Colour vision was tested using the FarnsworthMunsell 28-Hue test. ${ }^{9}$ Contrast sensitivity was evaluated with the Vistech Tables. ${ }^{10}$ Automated perimetry was performed using the central 24-2 program of the Humphrey field analyzer (model 630), with the foveal threshold option. ${ }^{11,12}$ Each patient had previous experience with automated perimetry. Flash visual evoked potentials (VEP) were recorded using the system of amplification, summation and recording system of the Conel SRL (Rome, Italy) instrument. Flash VEP was recorded by monopolar derivation with $\mathrm{Ag}-\mathrm{AgCl}$ cup electrodes placed in $\mathrm{Oz}$ (active) and $\mathrm{Fz}$ (reference) according to the 10/20 International System. The ground electrode was attached to the ear lobe. The intensity of the stimulus (white light flashes) was $1.5 \mathrm{~J}$ presented randomly at $1 \mathrm{~Hz}$. Filtering frequencies were $0.2-80 \mathrm{~Hz}$. The response to 100 light stimuli was averaged. ${ }^{13}$ Furthermore, the patients were advised to practise the apprehended techniques at home by trying to read the words on distance visual acuity charts with letters in a different order from those used to measure visual acuity, supplied by the examiner.

Successively, we extended the study enrolling eight patients (16 eyes) with bilateral low vision with the forementioned eligibility criteria. The patients were composed of one male and seven females with an age range of 23-86 years. Four patients had age-related macular degeneration, two patients had diffuse pallor of the optic nerve, one patient presented myopic maculopathy and one patient had Stargardt's macular degeneration.

In all patients training with Ibis was performed in only one eye with the procedure already described and the right eye was conventionally chosen for this purpose. The left eyes received a placebo training. Thus, during each session, after training of the right eye, the patient was asked to look with the left eye at the red light located in the occluder of the instrument.
This red light was set at the maximum level of frequency in order to be perceived as a fixed light.

In this part of the study two different examiners performed $\mathrm{BF}$ and measured visual acuity every three sessions. The number of words read per minute, colour vision test, contrast sensitivity test, automated perimetry and VEP were performed.

In the third part of the study only placebo training was performed, enrolling seven patients (12 eyes) with low vision with the same inclusion criteria as the forementioned cases. There were five bilateral and two unilateral cases. The patients were composed of two males and five females with age range of 29-76 years. Three patients (six eyes) had age-related macular degeneration, two patients (three eyes) presented myopic maculopathy and two patients (three eyes) had diffuse pallor of the optic nerve. The placebo training was developed to mimic the attention, equipment and time involved in the technique of biofeedback. During each session, the patient was asked to look at the red light located in the occluder of the instrument. This light, that could be used at different levels of frequency, was set at the maximum level in order to be perceived as a fixed red light. Similar to the experimental training, visual acuity was measured after every three sessions and a series of tests (number of words read per minute, colour vision test, contrast sensitivity test, automated perimetry, VEP) were carried out before and after having completed control training.

Intergroup differences were evaluated by means of repeated measures analysis of variance (ANOVA) for continuous variables. Significance level was set at $P<0.05$.

\section{Results}

In the preliminary study twelve patients (16 eyes) completed the training; one patient with bilateral low vision was unable to finish for family reasons and was excluded from the study.

Visual acuity was improved in all but one eye (Figure 2); even though only 10 patients reported subjective amelioration of visual capacity. The mean value of visual acuity before training was 0.12 (SD 0.12 ) and 0.28 (SD 0.17) at the last follow-up. Visual acuity was stable at 1 month after termination of training in all patients. At 6 months visual acuity was maintained in all but four eyes where regression occurred. There was a significant improvement in visual acuity for the whole sample $(F=47.98, \mathrm{df}=5$, $P<0.001$ ).

The number of words read per minute was also improved in all but one case (Figure 3). Prior to BF, 


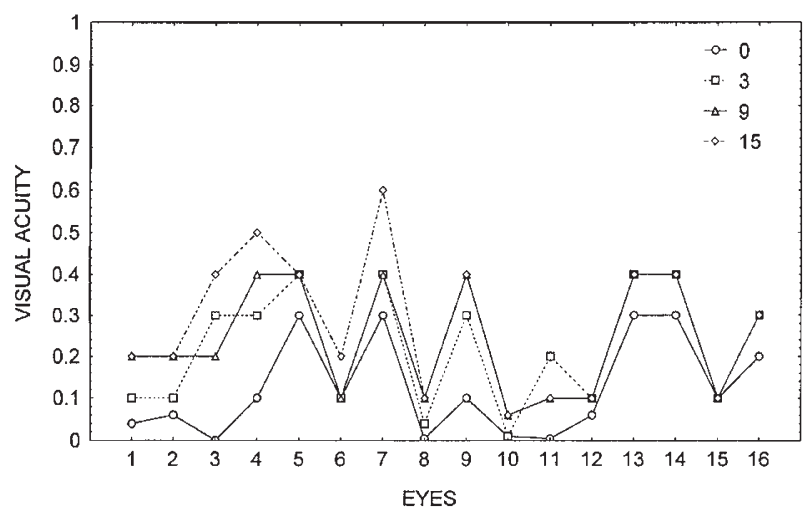

Figure 2 Visual acuity values for each eye before and after three, nine and 15 treatment sessions.

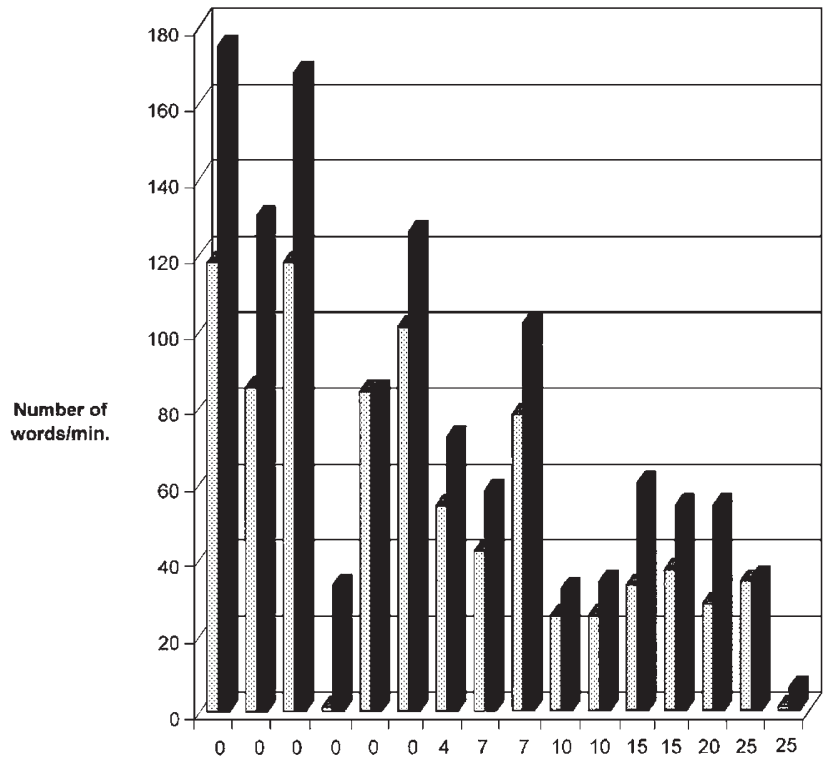

OBEFORE TREATMENT

- $A$ AFTER TREATMENT

Figure 3 Number of words read per minute/enlargement for each eye before and after treatment. No enlargement corresponds to ' 0 '.

colour vision was normal in six eyes, altered in the blue/yellow system in eight eyes and not measurable in two eyes. After treatment the alterations in the blue/yellow system were reduced in five eyes. Furthermore, the two patients who could not differentiate the colours managed, even though with numerous errors in the blue/yellow system, to carry out the test. Therefore, colour vision improved in $70 \%$ of cases and was unchanged in 30\%.

Contrast sensitivity testing and automated perimetry was only carried out in some cases as some patients were unable to perform these tests due to their low vision. In ten patients (14 eyes) where it was possible

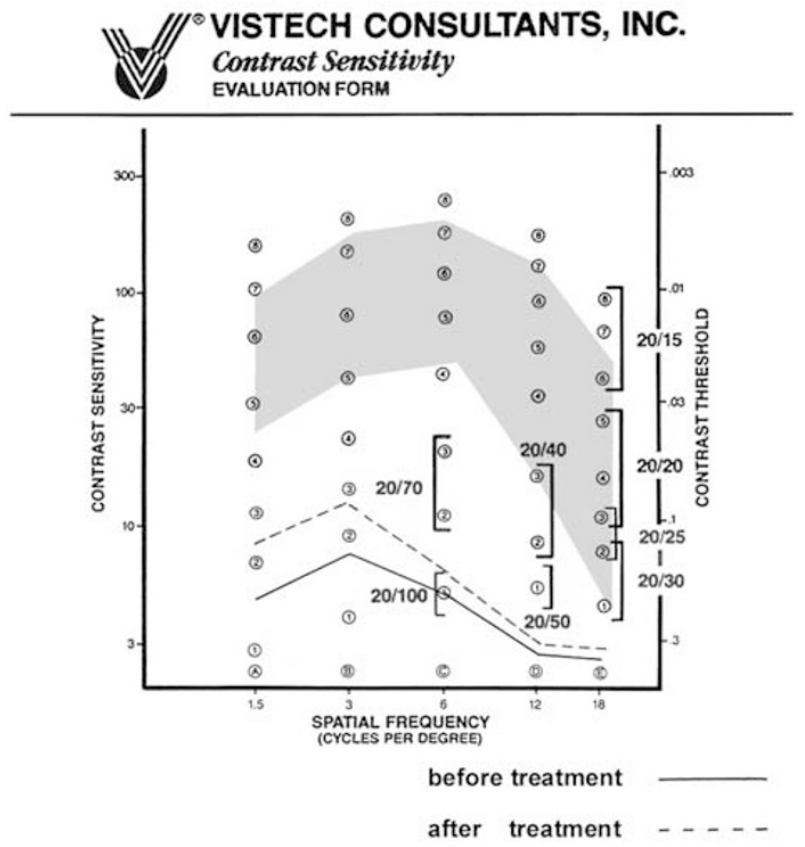

Figure 4 Mean values of contrast sensitivity before and after treatment.

to perform contrast sensitivity values were increased after treatment. The curves relative to pre- and posttraining mean values are shown in Figure 4.

It was possible to carry out visual field testing on 14 eyes. The mean values of foveal threshold, mean defect (MD), pattern standard deviation (PSD), correct pattern standard deviation (CPSD) and short fluctuation (SF) are reported in Table 1 .

Flash VEPs did not show significant variations either in morphology or in latency and amplitude. The mean latency of the P2 wave was $137.12 \mathrm{~ms}$ (SD 13) before and $137.06 \mathrm{~ms}$ (SD 14.19) after training. The mean amplitude of the P2 wave was $4.51 \mu \mathrm{V}$ (SD 1.26) before and $4.05 \mu \mathrm{V}$ (SD 1.24) after training.

The critical fusion frequency, shown by the instrument, had a mean increase from $21 \mathrm{~Hz}$ before to $26.45 \mathrm{~Hz}$ after training.

Table 1 Mean values $(\mathrm{db})$ of foveal threshold, mean defect (MD), pattern standard deviation (PSD), correct pattern standard deviation (CPSD) and short fluctuation (SF) using the Central 24-2 program of the Humphrey field analyzer (preliminary study)

\begin{tabular}{lcc}
\hline & Before treatment & After treatment \\
\hline Fovea & 18.83 & 20.83 \\
MD & 9.73 & 8.88 \\
PSD & 4.68 & 5.43 \\
CPSD & 4.82 & 5.46 \\
SF & 1.88 & 1.7 \\
\hline
\end{tabular}


In the second part of the study the results obtained for the right and left eyes were considered and analyzed separately. The data were then compared between the two groups.

Five of the eight eyes undergoing training with BF showed improvement of visual acuity. The mean value of visual acuity in the right eyes before treatment was 0.20 (SD 0.11), and 0.31 (SD 0.22) at the last follow-up. The visual acuity also improved in five of eight fellow eyes with an initial value of 0.16 (SD 0.11) to 0.27 (SD $0.24)$. The values relative to visual acuity are shown in Figure $5 \mathrm{a}$ and $\mathrm{b}$. On the whole, in four patients (eight eyes) visual acuity had bilateral improvement, in two patients there was improvement in only one eye (one right and one left eye) and in two patients there was no change. Four patients expressed subjective improvement of visual capacity. At 6 months from the termination of training sessions, visual acuity was stable in four right and four left eyes (four patients). The analysis showed a significant improvement in visual acuity in both the right $(F=16.46, \mathrm{df}=5$, $P<0.05)$ and left eyes $(F=18.22, \mathrm{df}=5, P<0.05)$.

The test to evaluate the number of words per minute was improved in six eyes (three right and three left eyes), was unchanged in nine eyes and was not
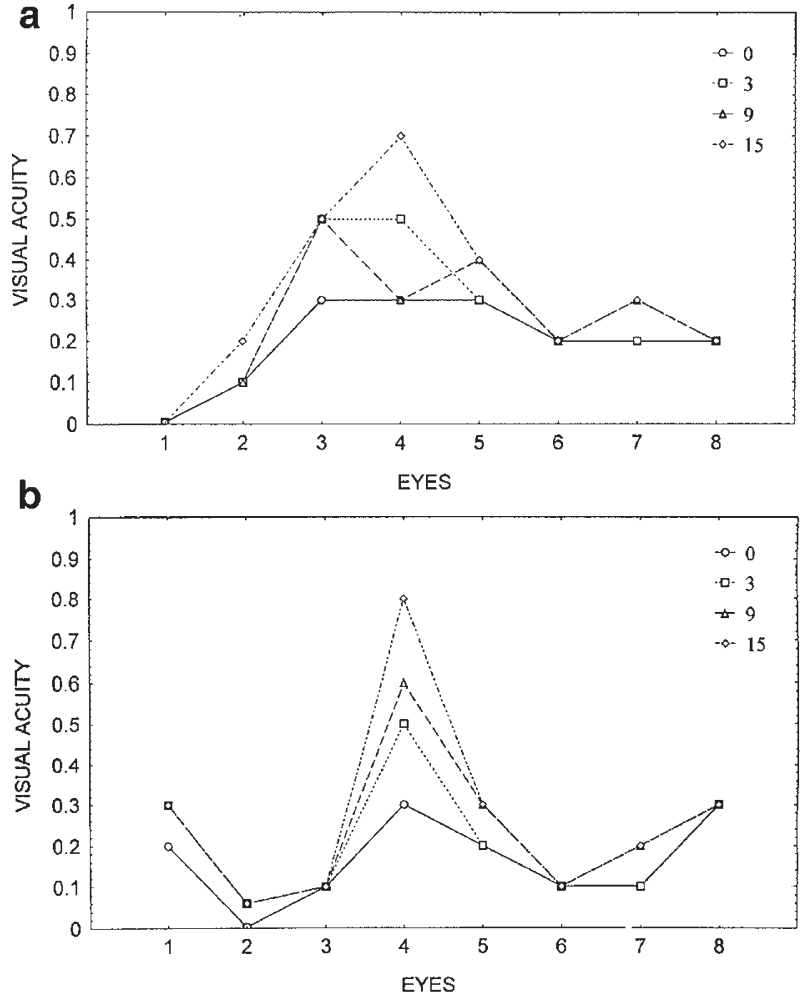

Figure 5 (a) Visual acuity values for right eyes before and after three, nine and 15 treatment sessions. (b) Visual acuity values for left eyes before and after three, nine and 15 treatment sessions. measurable in one (Figure 6a and b). Prior to training colour vision was normal in four eyes, altered for deficit in the blue/yellow system in 11 eyes and not measurable in one. At the end of BF sessions it was
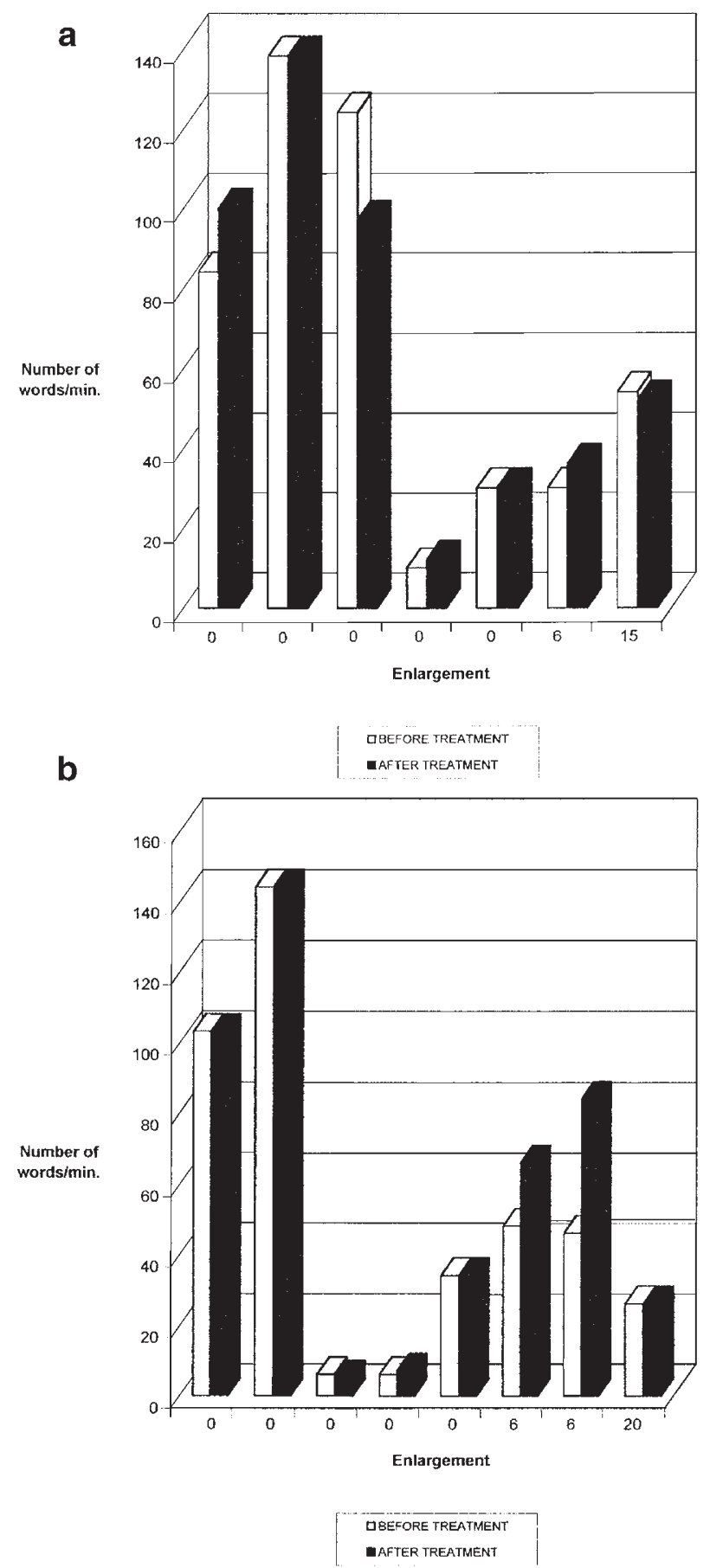

Figure 6 (a) Number of words read per minute/enlargement for each right eye before and after treatment. No enlargement corresponds to ' 0 '. (b) Number of words read per minute/ enlargement for each left eye before and after treatment. No enlargement corresponds to ' 0 '. 
improved in five patients (three right and two left eyes) and unchanged in all the rest.

The measurement of contrast sensitivity was possible in 15 eyes (seven right and eight left eyes). After termination of training the values were increased in three right and three left eyes and were not modified in the rest (Figure $7 a$ and $b$ ).

It was possible to perform visual field testing in all but one patient. The mean values of foveal threshold, mean defect (MD), pattern standard deviation (PSD), correct pattern standard deviation (CPSD) and short fluctuation (SF) are reported in Table 2.

Flash VEPs did not show significant changes in morphology, latency and amplitude after training. For the right eyes the mean latency of the P2 wave was $136.5 \mathrm{~ms}$ (SD 7.91) before and $136.25 \mathrm{~ms}$ (SD 6.94) after treatment and the mean amplitude was $4.01 \mu \mathrm{V}$ (SD 1.26) before and $4.26 \mu \mathrm{V}$ (SD 1.33) after treatment. For the left eyes the mean latency of the P2 wave was $135 \mathrm{~ms}$ (SD 5.47) before and $135.6 \mathrm{~ms}$ (SD 7.20) after treatment and the mean amplitude was $4.05 \mu \mathrm{V}$ (SD 1.64) before and $3.55 \mu \mathrm{V}$ (SD 0.95) after treatment. The critical fusion frequency shown by the instrument demonstrated a mean increase from $22.1 \mathrm{~Hz}$ before training to $26.71 \mathrm{~Hz}$ after training in the right eyes.

In the third part of the study visual acuity was improved by 0.1 in two eyes (Figure 8 ). The mean value of visual acuity before training was 0.19 (SD 0.10 ) and 0.21 (SD 0.12) at the last follow-up. Only one patient expressed subjective improvement of visual capacity. The improvement of visual acuity was not significant $(F=2, \mathrm{df}=1, P=0.15)$. The number of words read per minute was improved in two cases by one word (Figure 9). Prior to BF colour vision was normal in two eyes and altered in the blue/yellow system in 10 eyes. After placebo training it was improved in one eye. The values of contrast sensitivity were slightly increased in one eye (Figure 10).

The mean values of foveal threshold, mean defect (MD), pattern standard deviation (PSD), correct pattern standard deviation (CPSD) and short fluctuation (SF) are reported in Table 3.

Flash VEPs did not show significant changes either in morphology, or in latency and amplitude. The mean latency of the P1 wave was $134 \mathrm{~ms}$ (SD 6.92) before and 134.25 (SD 6.51) after training. The mean amplitude of the P2 was $4.65 \mu \mathrm{V}$ (SD 0.88) before and $4.3 \mu \mathrm{V}$ (SD 1.22) after placebo training.

There were no adverse effects in the three parts of the study.

\section{Discussion}

Techniques of BF have been performed in the treatment of ametropia (myopia, astigmatism, a

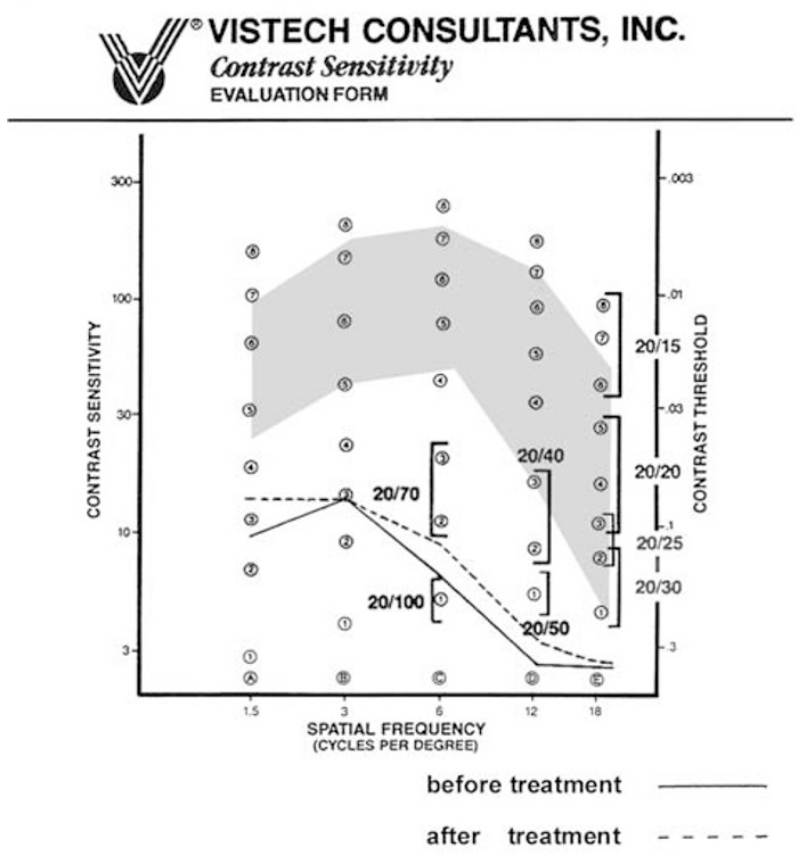

b
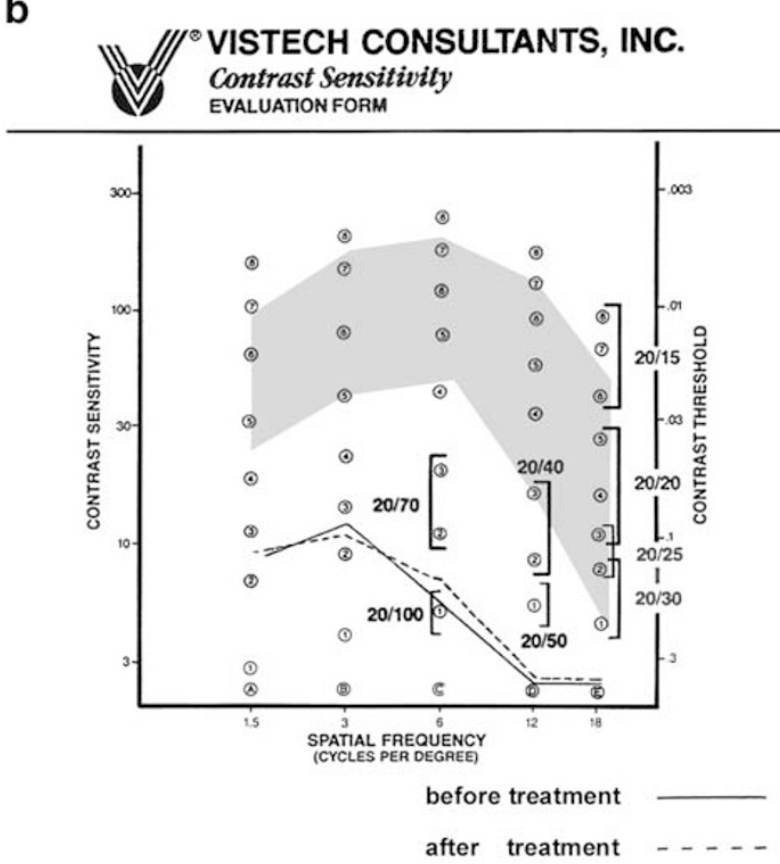

Figure 7 (a) Average values of contrast sensitivity for the right eyes before and after treatment. (b) Average values of contrast sensitivity for the left eyes before and after treatment.

presbyopia), nystagmus and amblyopia. ${ }^{1-7,14}$ The use of these methods in cases where conventional treatment cannot give further results presents an interesting alternative. Visual training may be considered as a 
Table 2 Mean values (db) of foveal threshold, mean defect (MD), pattern standard deviation (PSD), correct pattern standard deviation (CPSD) and short fluctuation (SF) using the Central 24-2 program of the Humphrey field analyzer

\begin{tabular}{lcclll}
\hline & \multicolumn{3}{c}{ Right eye } & & \multicolumn{2}{l}{ Left eye } \\
\cline { 2 - 3 } \cline { 5 - 6 } & $\begin{array}{l}\text { Before treat- } \\
\text { ment }\end{array}$ & $\begin{array}{l}\text { After treat- } \\
\text { ment }\end{array}$ & & $\begin{array}{l}\text { Before treat- } \\
\text { ment }\end{array}$ & $\begin{array}{l}\text { After } \\
\text { treatment }\end{array}$ \\
\hline Fovea & 26.20 & 27 & 22.50 & 23.16 \\
MD & 5.80 & 5.06 & & 13.14 & 12.06 \\
PSD & 5.46 & 5.23 & & 7.52 & 6.99 \\
CPSD & 4.83 & 4.83 & & 6.08 & 6.19 \\
SF & 1.94 & 1.66 & & 2.70 & 2.80 \\
\hline
\end{tabular}

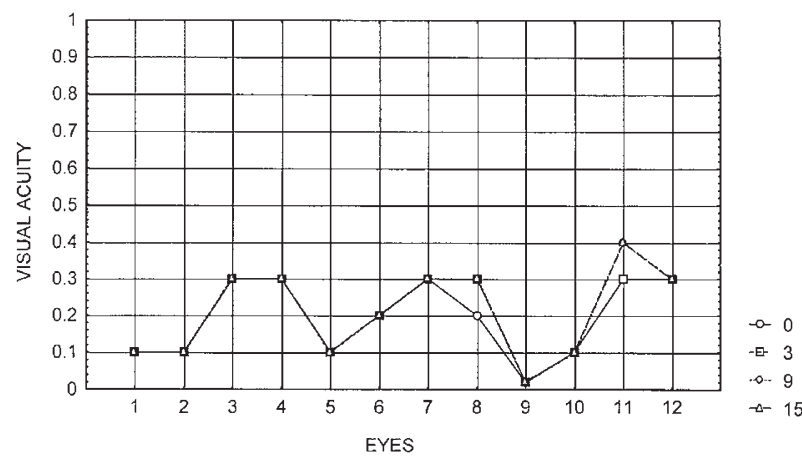

Figure 8 Visual acuity values for each eye before and after three, nine and 15 placebo treatment sessions.

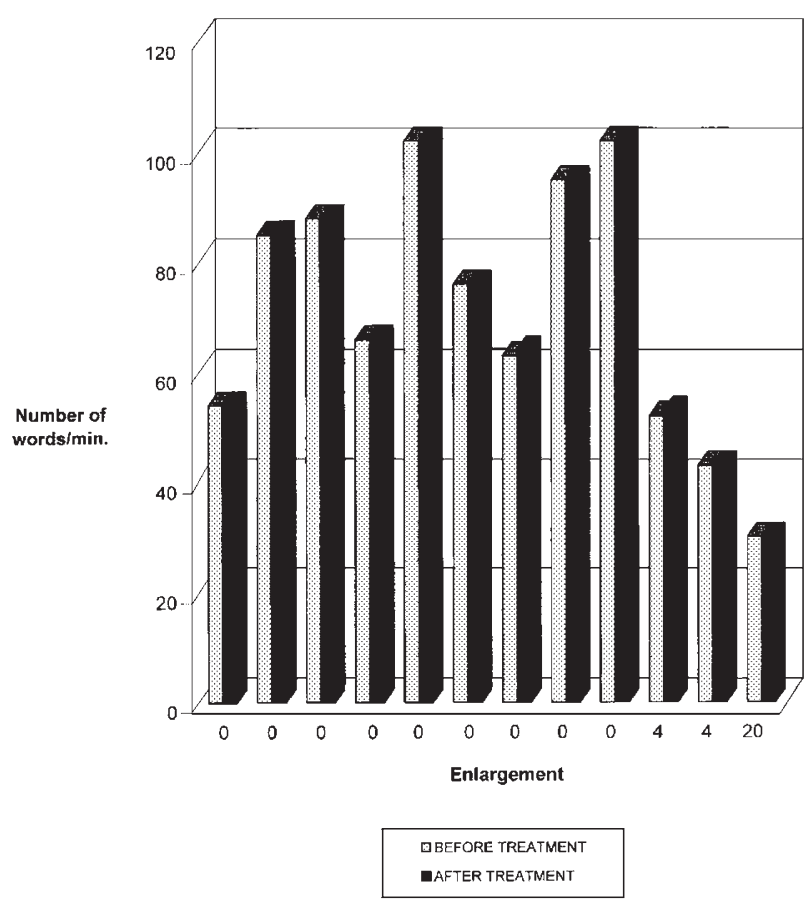

Figure 9 Number of words read per minute/enlargement for each eye before and after placebo treatment. No enlargement corresponds to ' 0 '.

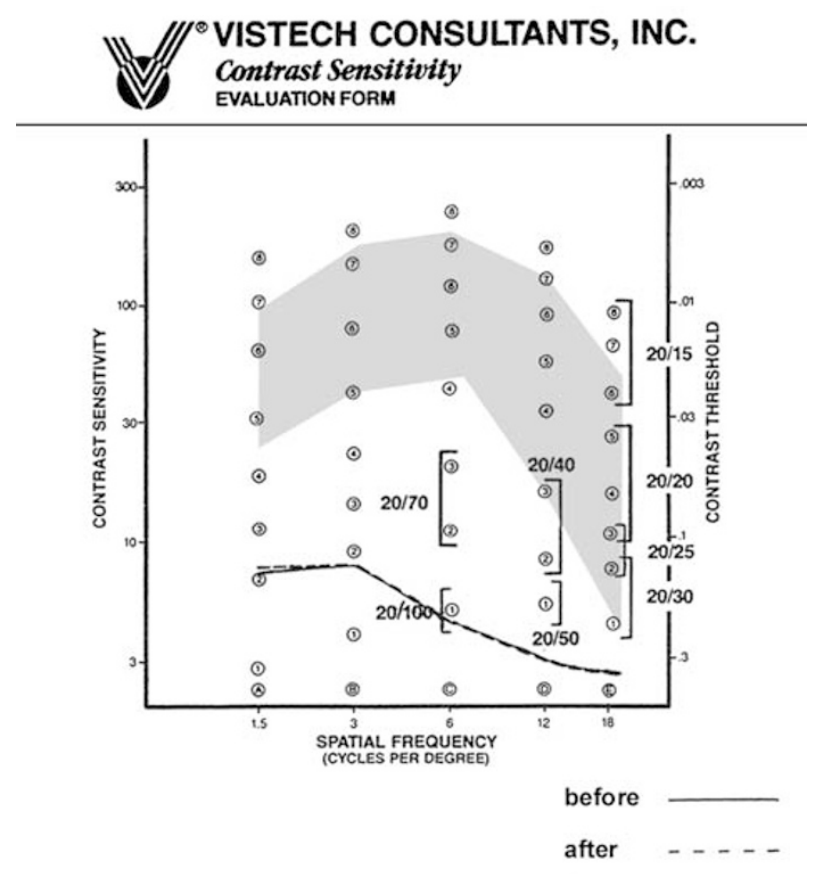

Figure 10 Mean values of contrast sensitivity before and after placebo treatment.

Table 3 Mean values $(\mathrm{db})$ of foveal threshold, mean defect (MD), pattern standard deviation (PSD), correct pattern standard deviation (CPSD) and short fluctuation (SF) using the Central 24-2 program of the Humphrey field analyzer (placebo treatment)

\begin{tabular}{lcc}
\hline & Before treatment & After treatment \\
\hline Fovea & 21.00 & 21.17 \\
MD & 7.58 & 7.49 \\
PSD & 5.56 & 5.71 \\
CPSD & 4.97 & 5.09 \\
SF & 1.62 & 1.78 \\
\hline
\end{tabular}

rehabilitative technique towards improving residual vision.

In the patients enrolled in the preliminary study visual acuity improved in all but one case after 15 sessions of training. Furthermore, the number of words read per minute was also improved in all but one case. It has been reported that patients with macular scotomas often develop a new area for fixation outside the altered macular area; it acts like a pseudo-fovea and is commonly called preferred retinal locus. ${ }^{15,16}$ Duret et $a l^{17}$ observed a combined use of several preferred retinal loci in patients with central scotomas when reading single words. In the present study the use of one or several preferred retinal loci may be considered. With biofeedback patients could become aware of their defect and learn to control their fixation pattern and their oculomotor behaviour. The highest 
level of BF, expressed by the increase in volume of the auditory signal, could be reached when the fixation was modified, even if the mechanism of action is not clear. When the patient has learnt, with BF, to modify oculomotor behaviour, this function is automatically controlled through practice.

It is well known that in age-related macular degeneration and in myopic degeneration the alterations of colour vision are in the blue/yellow system; whereas in optic nerve pathology these may be in the red/green or in the blue/yellow system. In the present study the alterations of colour vision were all in the blue/yellow system. It is significant that the two patients who could not differentiate the colours managed to carry out the test after training even though they made numerous errors in the blue/yellow system. Contrast sensitivity, in 14 eyes where it was possible to perform the test, was increased. It must be considered, however, that in the majority of these cases, only the orientation of some discs on the lines with lower spatial frequency was recognized because the visual acuity was low. At low spatial frequencies the image covers a portion of the retina that is much larger than the fovea and detection depends upon the function of the extramacular retina. At times, the patient's capability may be diminished below that which is expected from visual acuity as a result of additional low spatial frequency loss. ${ }^{18,19}$ Visual field testing performed in 14 eyes showed a mean increase of foveal threshold of $2 \mathrm{db}$. Since the foveal threshold is correlated well with visual acuity, this result could confirm the visual improvement which occurred in the patients in our study. Only minimal changes were observed in evaluating the global indices before and after BF sessions.

Flash VEP was performed because in the majority of patients visual acuity was considerably low and it was impossible to use pattern stimulation. The

characteristics of the P2 wave were studied as this is the only constant component and the other waves have high individual variability. The average changes in amplitude and latency observed after training were not significant.

Ibis is a new instrument which acts with various mechanisms. According to the manufacturers there could be activation of retinal function by infrared light resulting in $\mathrm{BF}$. The patient would monitor retinal activity by trying to increase the intensity of the return signal. The mechanism of infrared light on retinal processes remains obscure. Furthermore, Ibis has a system for passive foveal stimulation with variable intermittence in relationship to the individual critical fusion frequency. ${ }^{5}$ This method would improve visual function activating the on-off receptors which send a high quantity of macular stimuli to the visual cortex.

Various hypotheses regarding the mechanisms of visual function improvement after visual training techniques can be put forth. There could be improvement in ocular motor control and in 'searching capacity'. Furthermore, learning to use eccentric fixation could be a mechanism contributing to amelioration. ${ }^{14}$ Another suggestion is an increase in the discriminating capacities both of the retina and the visual cortex and associated areas. ${ }^{20}$ In our study we did not observe significant changes in flash VEP measures, but this test is not so sensitive as to evidence slight changes in visual function. In particular it is very important to note that visual function could be improved because patients undergoing training improve their ability to demonstrate their best visual acuity and other visual abilities.

The stability of results after 6 months in the majority of patients could depend on individual capacity in applying the techniques learnt through BF even in the absence of the acoustic return signal.

In the second part of the study visual acuity, number of words read per minute, colour vision, contrast sensitivity and visual field also improved in the majority of patients. Furthermore, visual acuity was stable at 6 months from the end of training in four patients. The response to $\mathrm{BF}$ was lower with respect to the first part of our study both regarding quantitative and qualitative aspects. This could be explained considering subjective variables such as intrinsic differences in response to training. Furthermore, two different examiners performed the sessions of $\mathrm{BF}$ and measured visual acuity; a different patient/therapist relationship could have influenced the results. Indeed in this part of our study, in order to avoid patient conditioning and misinterpretation of results, the examiner who carried out the sessions of BF did not perform visual acuity measurement. In the preliminary part of our research the examiner both performed training and measured visual acuity. Therefore, even though influencing of 'pre' and 'post' results was avoided regarding the choice of difficult letters and the time given for patient response, there still could have been some influence (examiner effect).

The rather unexpected result in the second part of the study was, however, a similar improvement of visual acuity and functional test results in the controlateral eye to training. This may be explained as both due to training of the patient and to monocular perceptive visual learning where there are effects also on the fellow eye, as the site of learning would be the cortex. ${ }^{21}$ Animal model experiments have demonstrated the existence of flexibility of the adult visual system 
through which after retinal lesions there are modifications of the neuronal receptor fields of the visual cortex. ${ }^{22}$ Furthermore, there could be a possible role of conjugate eye movements which arise following visual, acoustic or other types of stimuli. ${ }^{23}$

In the third part of the study visual acuity was slightly improved in only two eyes and statistical analysis was not significant. The number of words read per minute, colour vision and contrast sensitivity testing showed negligible variations. The results obtained for visual field testing and flash VEPs did not show significant changes.

Surely a certain role in the determination of the results could be due to subjective variables such as learning effect, motivation, level of attention, psychophysical capacities and influence of the examiner.

At present it is not yet possible to advance a valid scientific theory on the true psycho-physiological mechanisms of action which make training with Ibis and other methods of biofeedback useful. Our results undoubtedly show a rather unexpected amelioration in visual acuity and the majority of the diagnostic tests performed.

Improvement through BF training in patients who are afflicted with pathologies which remain either stable or worsen, where traditional treatment cannot offer further results, is of interest and well worthy of attention. Further study in this field is warranted.

\section{Acknowledgements}

The authors have no proprietary interest in the materials and methods reported in this manuscript.

\section{References}

1 Trachtman JN. Biofeedback of accomodation to reduce myopia. A case report. Am J Optom Physiol Opt 1978; 55: 400-406.

2 Abadi RV, Carden D, Simpson J. A new treatment for congenital nystagmus. Br J Ophthalmol 1980; 64: 2-6.

3 Ciuffreda KJ, Goldrich SG, Neary C. Use of eye movement auditory feedback in the control of nystagmus. Am J Optom Physiol Opt 1982; 59: 396-409.

4 Kirschen DG. Auditory feedback in the control of congenital nystagmus. Am J Optom Physiol Opt 1983; 60: 364-368.

5 Fossetti A. The management of myopia by biofeedback visual training. International Conference of Optometry, Riga, 1962.

6 Moncada A. Il Biofeedback Visivo: Origini, Ipotesi Fisiologiche, Applicazioni. Atti SIRV: Roma, Italy, 1995, pp 6-11.

7 Leung V, Wick B, Bedell HE. Multifaceted treatment of congenital nystagmus: a report of 6 cases. Optom Vision Sci 1996; 73: 114-124.

8 Iorio P, Albergo V, Bianco G, Spadea L, Rapinese M, Gallenga PE, Balestrazzi E. Fotostimolazione visiva e ipovisione. Ottica fisiopatologia 1998; 3: 39-44.

9 Farnsworth D. The Farnsworth-Munseel 100-hue and dichotomous test for color vision. J Opt Soc Ann 1943; 33: 568-578.

10 Ginsburg AP. A new contrast sensitivity test chart. Am J Optom Physiol Opt 1984; 61: 403-407.

11 Heijl A. The Humphrey field analyzer, construction and concepts. Doc Ophthalmol Proc Series 1985; 42: 77-84.

12 Haley MJ. The Field Analyzer Primer, 2nd edn. Allergan Humphrey: San Leandro, CA, 1987.

13 Contestabile MT, Suppressa F, Tonelli G, Giorgi D, Antonnicola G, D'alba E. The influence of age on the flash visual evoked potentials. Acta Ophthalmol Scand 1995; 73: 308-312.

14 Trachtman JN. Raccolta dei Risultati Ottenuti in Pazienti a Seguito del Trattamento con l'Allenatore Della Vista Accomotrack Vision Trainer-Riduzione Della Miopia. Atti del XVI corso di aggiornamento Apimo. Montecatini Terme: Italy, 1994, pp 183-195.

15 Von Noorden GK, Mackensen G. Phenomenology of eccentric fixation. Am J Ophthalmol 1962; 53: 642.

16 Cummings RW, Whittaker SG, Watson GR, Budd JM. Scanning characters and reading with a central scotoma. Am J Optom Physiol Optics 1985; 62: 833-843.

17 Duret F, Issenhuth M, Safran AB. Combined use of several preferred retinal loci in patients with macular disorders when reading single words. Vision Res 1999; 39: 873-879.

18 Loshin DS, White J. Contrast sensitivity: the visual rehabilitation of the patient with macular degeneration. Arch Ophthalmol 1984; 102: 1303-1306.

19 Fleishman JA, Beck RW, Linares DA et al. Deficits in visual function after recovery from optic neuritis. Ophthalmology 1987; 94: 1029-1035.

20 D'Andrea P. Risultati della Rieducazione Visiva in un Caso di Maculopatia; Valutazioni Campimetriche. Atti SIRV, Roma, Italy, 1995, pp 37-46.

21 Fiorentini A, Berardi N. Visual perceptual learning. A sign of neural plasticity at early stages of visual processing. Archives Italiennes de Biologie 1997; 135: 156167.

22 Maffei L, Fiorentini A. The unresponsive regions of visual cortical receptive fields. Vision Res 1976; 16: 1131-1139.

23 Diversi A, La Rosa G, Liuzzi L. Lo Strabismo Concomitante. Edizioni Libreria Cortina. Tipolitografia Stampart: Torino, 1985, p 32 